\title{
miR-224 is Critical for Celastrol-Induced Inhibition of Migration and Invasion of Hepatocellular Carcinoma Cells
}

\author{
Hangyu Lia Yan Lia Dan Liu ${ }^{a}$ Hongzhi Sun ${ }^{b}$ Fuquan Yang ${ }^{a}$ Jingang Liu ${ }^{a}$
}

aDepartment of General Surgery, Shengjing Hospital Affiliated to China Medical University, China Medical University, Shenyang; ${ }^{b}$ Department of General Surgery, First Hospital Affiliated to Liaoning Medical College, Jinzhou

\section{Key Words}

Migration and invasion $\cdot \operatorname{miR}-224 \cdot$ Celastrol $\cdot N F-k B \cdot M M P s$

\begin{abstract}
Background/Aims: The molecular mechanisms of celastrol on hepatocellular carcinoma (HCC) cells migration and invasion ability is the major problem that prompted the study. Methods: We first evaluated the effect of celastrol on migration and invasion ability of HepG2 cells using transwell migration and matrigel invasion assays. Next, we assessed the effect of celastrol on NF-KB transcriptional activity in hepatocellular carcinoma cells using western blot and luciferase reporter assay. We also performed real-time PCR to measure miR-224, MMP-2 and MMP-9 expression. Western blot was used to measure protein expression of MMP-2 and MMP-9. Furthermore, we used miR-224 inhibitor to evaluate whether down-regulation of miR224 expression can affect MMP-2 and MMP-9 expression. The binding ability of p65/NF- $\mathrm{kB}$ on the miR-224 promote has been assessed by chromatin immunoprecipitation and quantitative real-time PCR (ChIP-qPCR). Finally, we evaluated the effect of miR-224 on celastrol-induced anti-tumor activity using miR-224 precursor. Results: Celastrol significantly impaired migration and invasion of HepG2 cells and inhibited the activation of NF-kB and Akt in dose-dependent manner. IGF (the strong stimulator of Akt) inhibited the transcriptional activity of NF-KB in cells treated with celastrol. Besides, celastrol efficiently decreased the expression of miR-224 and protein expression of MMP-2 and MMP-9. ChIP-qPCR showed that p65/NF-kB binding to the miR-224 promoter sharply decreased after exposure to celastrol in time-dependent manner. Furthermore, inhibition of miR-224 expression can decrease MMP-9 protein level. Most importantly, miR-224 precursor can reverse the effect of celastrol on impairment of migration and invasion in HepG2 and Huh7 cells. Conclusion: Celastrol treatment inhibits migration and invasion of $\mathrm{HCC}$ cell and that the effect is partly due to NF-KB regulating miR-224 expression.
\end{abstract}

Copyright (C) 2013 S. Karger AG, Basel 
Li/Li/Liu/Sun/Yang/Liu : Celastrol on Migration and Invasion of HCC Cells

\section{Introduction}

Hepatocellular carcinoma (HCC) is one of the most common malignant tumors worldwide, particularly high in East Asia and sub-Saharan Africa [1]. One of characteristics of HCC is vascular invasion propensity and high metastatic potential, which becomes the greatest barrier to cancer cure and is the prime reason for cancer patient mortality [2]. Tumor cells invasion and metastasis are complicated processes involving dys-regulation of molecular signal pathways and proteins $[3,4]$. Therefore, it is urgent to make clear the key molecular mechanism of the metastasis in HCC as well as establishment of the appropriate blocking channels is important for the development of more effective therapies of HCC.

MicroRNAs (miRNAs) are a new class of endogenous, non-coding RNAs involved in modulating gene expression [5]. miRNAs are implicated in a wide range of physiological processes, such as development, apoptosis and differentiation [6]. However, miRNAs are aberrantly expressed in cancer and act as tumor suppressors or oncogenes [7]. In recent years, miRNAs were found to play an important role in invasion and metastasis of several tumors, such as breast, liver, prostate and colorectal cancers [8]. One of these miRNAs, miR224 is over-expressed in HCC and involved in cell proliferation, migration and invasion [9]. When the expression of miR-224 was down-regulated in HepG2, it was showed to inhibit cell migration and invasion [10]. Scisciani et al reported that NF- $\kappa B$ was a direct transcriptional regulator of miR-224 expression and linked cell migration and invasion in HCC [11].

Celastrol is a plant triterpene derived from the root of Thunder of God Vine and has been shown to possess anti-inflammatory and anti-cancer properties [12-14]. It has been reported that celastrol could inhibit proliferation and induce apoptosis of wide variety of human tumor cell types including multiple myeloma, hepatocellular carcinoma, gastric cancer and so on [15]. Besides, celastrol exerts potent antimetastatic activity by modulating migration and adhesion in lung cancer cells, melanoma cells and breast cancer cells [16, 17]. However, there is no available information for the molecular mechanism of the antimigration and anti-invasion functions of celastrol on HCC cells. Therefore, in the present study, we have investigated the effect of celastrol on migration and invasion in HCC cells and the underlying mechanisms associated with this activity.

\section{Materials and Methods}

\section{Chemicals and reagents}

DMEM was obtained from GIBCO (Invitrogen Company). Newborn calf serum (NCS) was from PAA Laboratories (GmbH, Linz, Austria). Celastrol was purchased from Alexis Biochemicals (San Diego, CA). Cytomatrix human vitronectin-coated strips were obtained from Chemicon International Inc (California, USA). MMP-9 ELISA kit was from R\&D (Abingdon, UK). Mouse anti-phospho-IкB (Ser32/36), rabbit anti$\mathrm{I} \kappa \mathrm{B}$ antibodies, rabbit polyclonal antibodies against phosphorylated Akt and total Akt were obtained from Cell Signaling Technology (Beverly, MA, USA). Polyclonal antibody against $\beta$-actin, p65, Lamin B MMP-2 and MMP-9 was purchased from Santa Cruz Biotechnology (Santa Cruz, CA, USA). The Detergent Compatible (DC) Protein Assay kit was purchased from Bio-Rad Laboratories (Hercules, CA, USA). The miRNeasy Mini kit, the miScript Reverse Transcription kit and the miScript SYBR Green PCR kit were purchased from Qiagen (Hilden, Germany).

\section{Cell culture}

Human hepatoma cell lines HepG2 and Huh7 were obtained from the American Type Culture Collection (Manassas, VA). The cells were cultured in DMEM, adjusted to contain $4 \mathrm{mM}$ L-glutamine, $1.5 \mathrm{~g} / \mathrm{l}$ sodium bicarbonate, $4.5 \mathrm{~g} / \mathrm{l}$ glucose, $10 \% \mathrm{FBS}, 100 \mathrm{units} / \mathrm{ml}$ penicillin, and $65 \mathrm{units} / \mathrm{ml}$ streptomycin. All cell lines were maintained at $37^{\circ} \mathrm{C}$ in a humidified incubator containing $5 \% \mathrm{CO}_{2}$. Cultured cells were treated with celastrol (dissolved in DMSO) in complete DMEM medium. To obtain reliable results, the final concentration of DMSO in the culture medium was kept less than $0.1 \%$. 
Li/Li/Liu/Sun/Yang/Liu : Celastrol on Migration and Invasion of HCC Cells

MTT assay

Cell viability was determined using MTT [3-(4,5-dimethylthiazol-2-yl)-2,5-diphenyltetrazolium bromide assays. Briefly, the cells were seeded in 96-well dishes at $1 \times 10^{4}$ cells per well, and treated with different concentrations of celastrol. Then each well was supplemented with $10 \mu \mathrm{L}$ MTT (Sigma Aldrich) and incubated for $4 \mathrm{~h}$ at $37^{\circ} \mathrm{C}$. The medium was then removed, and $150 \mu \mathrm{L}$ DMSO (Sigma Aldrich) was added to solubilize the MTT formazan. The optical density was read at $490 \mathrm{~nm}$.

\section{Flow cytometry analysis}

Flow cytometry analysis is based on the translocation of phosphatidylserine from the inner leaflet of the plasma membrane to the cell surface in early apoptotic cells [18]. Briefly, cells were resuspended in a binding buffer. Next, annexin V-EGFP and PI were added and the solution was incubated at room temperature for $15 \mathrm{~min}$ in the dark, followed by assay on FACScan (Becton Dickin-son). The percentage of apoptosis was computed using Cell-Quest software (Becton Dickinson).

\section{Transwell cell migration and matrigel invasion assays}

For Transwell migration assays, the cells were counted and $2 \times 10^{5}$ cells in $500 \mu \mathrm{L}$ serum-free DMEM containing the indicated concentration of celastrol were seeded into the upper part of each chamber, whereas the lower compartments were filled with DMEM supplemented with $10 \%$ fetal bovine serum as a chemo-attractant. For invasion assays, cells were plated in the top chamber with matrigel-coated membrane (8- $\mu$ m pore size, BD Biosciences, USA) where the transwell membrane was coated either with matrigel (100 $\mu \mathrm{g} / \mathrm{cm}^{2}$ ), while other methods were similar to the Transwell migration assays. Following incubation for 18 hours at $37^{\circ} \mathrm{C}$, noninvaded cells on the upper surface of the filter were wiped out with a cotton swab. The invaded cells on the lower surface of the filter were fixed formaldehyde (4\%) and stained with $0.1 \%$ crystal violet in $2 \%$ methanol. Invasiveness was determined by counting cells in five microscopic fields per well, and the extent of invasion was expressed as an average number of cells per microscopic field.

Quantitative real-time PCR (Q-PCR) analysis

Approximately $5 \times 10^{6}$ cells were treated without (Control) or with celastrol for $16 \mathrm{~h}$. miRNAs were isolated and purified using Trizol reagent (Invitrogen, USA), according to manufacturer's protocol. The miR-224 level was quantified by real-time PCR using TransStartTM SYBR Green qPCR Supermix (TransGen Biotech, Beijing, China), and with U6 small nuclear RNA as an internal normalized reference. For miR-224, the primers were as follows: forward, 5'-CAC TAG TGG TTC CGT TTA GTA G-3' and reverse, 5'-TTG TAG TCA CTA GGG CAC C-3'.

The mRNA expression of MMP-2 and MMP-9 was performed using SYBR GREEN PCR Master Mix (Applied Biosystems). The specific primers were as follows: MMP-2, 5'-ACT GTT GGT GGG AAC TCA GAA G-3' (forward) and 5'-CAA GGT CAA TGT CAG GAG AGG-3' (reverse); MMP-9, 5-TGG GTG TAC GAC GGT GAA AA-3' (forward) 5'-CAT GGG TCT CTA GCC TGA TA-3' (reverse).

\section{Western blot analysis}

HepG2 and Huh7 cells were lysed with ice-cold lysis buffer containing: $50 \mathrm{mmol} / \mathrm{l}$ Tris-HCl, pH 7.4; 1\% NP-40; $150 \mathrm{mmol} / \mathrm{l} \mathrm{NaCl} ; 1 \mathrm{mmol} / \mathrm{l}$ EDTA; $1 \mathrm{mmol} / \mathrm{l}$ phenylmethylsulfonyl fluoride; and complete proteinase inhibitor mixture (one tablet per $10 \mathrm{ml}$; Roche Molecular Biochemicals, Indianapolis, IN, USA). Protein concentration in the cell lysate was quantified using the DC protein assay kit (Bio-Rad). After protein content determination using a DC Protein Assay kit, Western blot analysis was performed.

\section{Luciferase reporter assay}

The reporter plasmid, pNF- $\kappa B$-luc, containing the $\kappa B$-enhancer consensus sequences [(TGGGGACTTTCCGC) $\times 5$ ] and NF-кB-dependent firefly luciferase gene was purchased from Stratagene (La Jolla, CA, USA). HepG2 cells were transiently transfected with two plasmids (pNF-кB-luc plasmid and $\beta$-galactosidase) using the LipofectAMINE Plus regent. Cells were passaged on 24-wells plate the day prior to transfection to achieve $80 \%-85 \%$ confluence on the following day. Twenty-four hours after transfection, cells were incubated for an additional $12 \mathrm{~h}$ in medium containing different concentration of celastrol and harvested for luciferase reporter assays. Luciferase activity was measured with a luminometer (TD-20/20; 
Li/Li/Liu/Sun/Yang/Liu : Celastrol on Migration and Invasion of HCC Cells

Turner Designs, CA, USA) using the Luciferase Assay System. $\beta$-Galactosidase activity was detected to normalise any variations in the transfection efficiency.

Oligonucleotides and Cell Transfection

miR-224 was knocked down or overexpressed by transfection with miR-224 inhibitor or miR-224 precursor using siPort Neo-FX (Ambion) according to the manufacturer's recommendations. miR-224 precursor (5'- CAA CUC ACU AGU GGU UCC GUU3-3'), miR-224 inhibitor (5'- AAC GGA ACC ACU AGU GAC UUG-3-3') and negative control (NC, 5'-CAG UAC UUU UGU GUA GUA CAA-3') were synthesized by RIBOBIO (Ribobio Co. Ltd, Guangzhou, China). All of the oligonucleotides were transfected at a final concentration of $100 \mathrm{nM}$. HepG2 cells were transfected with miR-224a inhibitor or precursor using siPort Neo-FX (Ambion) according to the manufacturer's recommendations.

Chromatin immunoprecipitation and quantitative real-time PCR (ChIP-qPCR)

It has been reported that NF- $\mathrm{KB}$ could bind to the miR-224 promoter [11]. ChIP-qPCR assay were used to examine whether celastrol could affect the binding activity of P65/ NF- $\mathrm{KB}$ on miR-224 regulatory region ( -600 to $-400 \mathrm{bp}$ ). HepG2 cells were seeded in 10-cm plates and treated with celastrol for $1 \mathrm{~h}, 3 \mathrm{~h}$ or $12 \mathrm{~h}$. ChIP assay was performed as previously described [19]. The relative proportions of co-immunoprecipitated DNA fragments were determined based on the threshold cycle (Ct) value for each PCR reaction [20]. For every promoter studied, a Ct value was calculated for each sample: $\Delta \mathrm{Ct}=\mathrm{Ct}$ (sample)-Ct (Input). Next, a $\Delta \Delta \mathrm{Ct}$ value was calculated: $\Delta \Delta \mathrm{Ct}=\Delta \mathrm{Ct}$ (sample immunoprecipitated with P65 antibody) $-\Delta \mathrm{Ct}$ (sample immunoprecipitated with IgG). The fold difference between specific antibody-immunoprecipitated samples and those immunoprecipitated with IgG was thus calculated using the formula: $2^{-\Delta C \mathrm{Ct}}$. Sequences of the primers were 5'-TGT GCT GGT TTT CTG AAG TT-3' (forward) and 5'-ACC TGA TGC CTC TCA CGA CC-3' (reverse).

\section{Statistical analysis}

Statistical analysis was performed with statistical analysis software SPSS 13.0 software. Statistical analyses were performed using either an analysis of variance (ANOVA) or Student's t-test. Results are presented as means \pm SD. A P value $<0.05$ was deemed significant. A P value $<0.01$ was deemed highly significant.

\section{Results}

The effects of celastrol on the migration and invasion ability of HCC cells

It has been reported that celastrol is involved in HCC cell growth [21]. To investigate the pharmacological potential of celastrol in HCC, we first examined the effect of celastrol on cell growth in HepG2. HepG2 cells were treated with various concentrations of celastrol for $36 \mathrm{~h}$, and determined cell viability using MTT assay. Celastrol significantly decreased cell viability at a concentration of $2 \mu \mathrm{M}$ (Fig. 1A). We further explored the effect of celastrol on apoptosis of HepG 2 cells using flow cytometry analysis. Celastrol did not increase the number of cells that underwent apoptosis (early apoptosis, annexinV+ / PI- and late apoptosis, annexin V+ / PI+) at low dosage. However, a high dosage of celastrol $(2$ and $5 \mu \mathrm{M})$ triggered apoptosis of HepG2 cells (Fig. 1B). Next, we found that HepG2 cells treated with celastrol at low dosage $(0.1$ and $0.5 \mu \mathrm{M})$ significantly inhibited migration and invasion (Fig. 1C and D).

\section{The effects of celastrol on PI3K/Akt and NF- $\kappa B$ activity in HCC cells}

It is well known that NF- $\mathrm{BB}$ signaling pathway plays an important role in HCC metastasis [22]. Next, we wanted to determine whether NF- $\mathrm{KB}$ activity was inhibited by celastrol in HCC cells. Therefore, phosphorylation of ІкB was investigated as a potential response to treatment with celastrol. As shown in Fig. 2A, celastrol inhibited phosphorylation of IкB in a dose-dependent manner in HepG2 cells. However, celastrol had no effect on total IкB expression. 
Fig. 1. Celastrol repressed the migration and invasion ability of HCC cells viability. (A) HepG2 cells were incubated with the indicated concentration of celastrol for $36 \mathrm{~h}$, and the cell viability was measured using a MTT assay. ${ }^{*} \mathrm{p}<0.05$, versus vehicle alonetreated cells. (B) Flow cytometry analysis showed that celastrol induced apoptosis of HepG2 cells in dose-dependent manner. ${ }^{*} \mathrm{p}<0.05$, versus vehicle alonetreated cells. (C) Exposure to various concentrations of celastrol resulted in dosedependent migration inhibition of HepG2 cells. $* \mathrm{p}<0.05$, versus
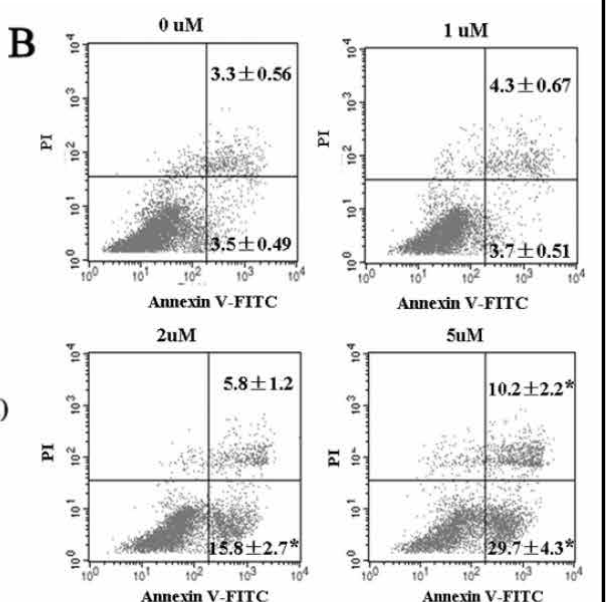

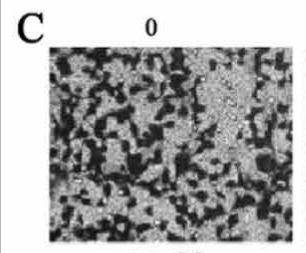

$0.5 \mu \mathrm{M}$

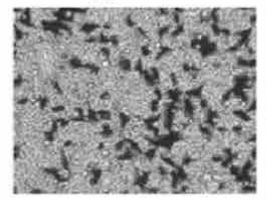

D

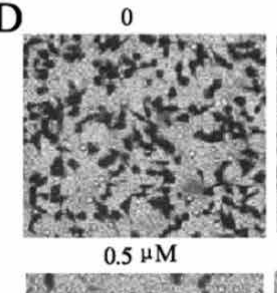

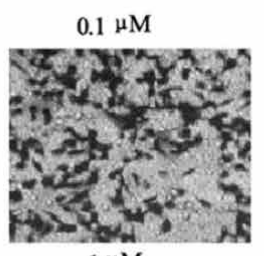

$1 \mu \mathrm{M}$

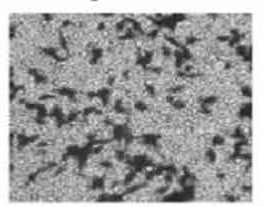

$0.1 \mu \mathrm{M}$

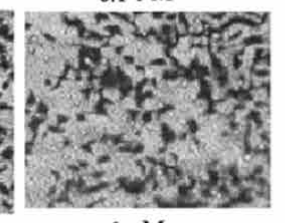

$1 \mu \mathrm{M}$

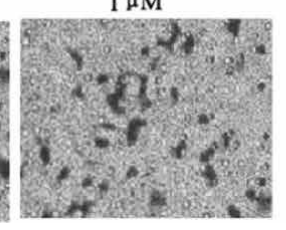

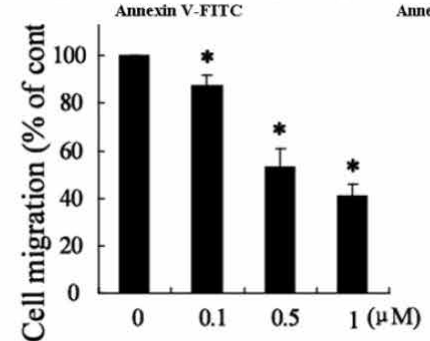

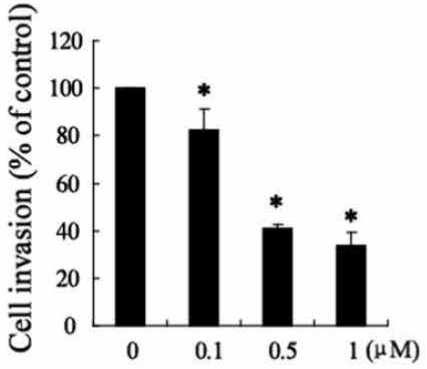

vehicle alone-treated cells. (D) Exposure to various concentrations of celastrol resulted in dose-dependent invasion inhibition of HepG2 cells. ${ }^{*} \mathrm{p}<0.05$, versus vehicle alone-treated cells.

To investigate the nuclear entry of NF- $\mathrm{BB}$ complex, the p65 content was measured by western blot in HepG2 cells. Treatment with celastrol $(0.5 \mu \mathrm{M})$ could inhibit p65 entry into the nucleus (Fig.2A). The inhibition of NF- $\kappa B$ signaling pathway by celastrol was also determined by the observation of the transcriptional activity of NF- $\mathrm{KB}$. As shown in Fig. 2B, the treatment of HepG2 cells with celastrol strongly inhibited the NF- $\kappa B$ transcriptional activity in a dose-dependent manner.

To explore whether PI3K/Akt signaling pathway was involved in the inhibition of NF$\kappa \mathrm{B}$ activity, we determined the phosphorylation of Akt and total Akt expression in HepG2 cells after treatment with celastrol. As shown in Fig. 2C, celastrol inhibited phosphorylation of Akt in a dose-dependent manner but had no effect on total Akt expression. Besides, cells were pre-treated with IGF $(100 \mathrm{ng} / \mathrm{ml}$, the strong stimulator of Akt) for $1 \mathrm{~h}$ and then exposed to celastrol. We found that IGF pretreatment blocked the decreased NF- $\kappa \mathrm{B}$ transcriptional activity by celastrol $(0.5 \mu \mathrm{M})$ as compared to control cells (Fig. 2D). Taken together, these results confirmed that celastrol decreased NF- $\kappa B$ activity via inhibition of the PI3K/Akt pathway. 
Fig. 2. Celastrol inhibited $N F-\kappa B$ signaling pathway in HCC cells. (A) Celastrol inhibited phosphorylation of I $\kappa \mathrm{B}$ in dose-dependent manner. Cytoplasmic P65 content was increased but nuclear p65 content was decreased in cells treated with celastrol. (B) Celastrol decreased $\mathrm{NF}-\kappa \mathrm{B}$ transcriptional activity in HepG2 cells in a dose-dependent manner. ${ }^{*} p<0.05$, versus vehicle alone-treated cells. (C) Celastrol inhibited phosphorylation of Akt in dose-dependent manner. (D) IGF greatly enhanced NF- $\mathrm{BB}$ transcriptional activity in HepG2 cells after treatment with celastrol. ${ }^{*} \mathrm{P}<0.05$, indicate significant differences from the respective control groups.
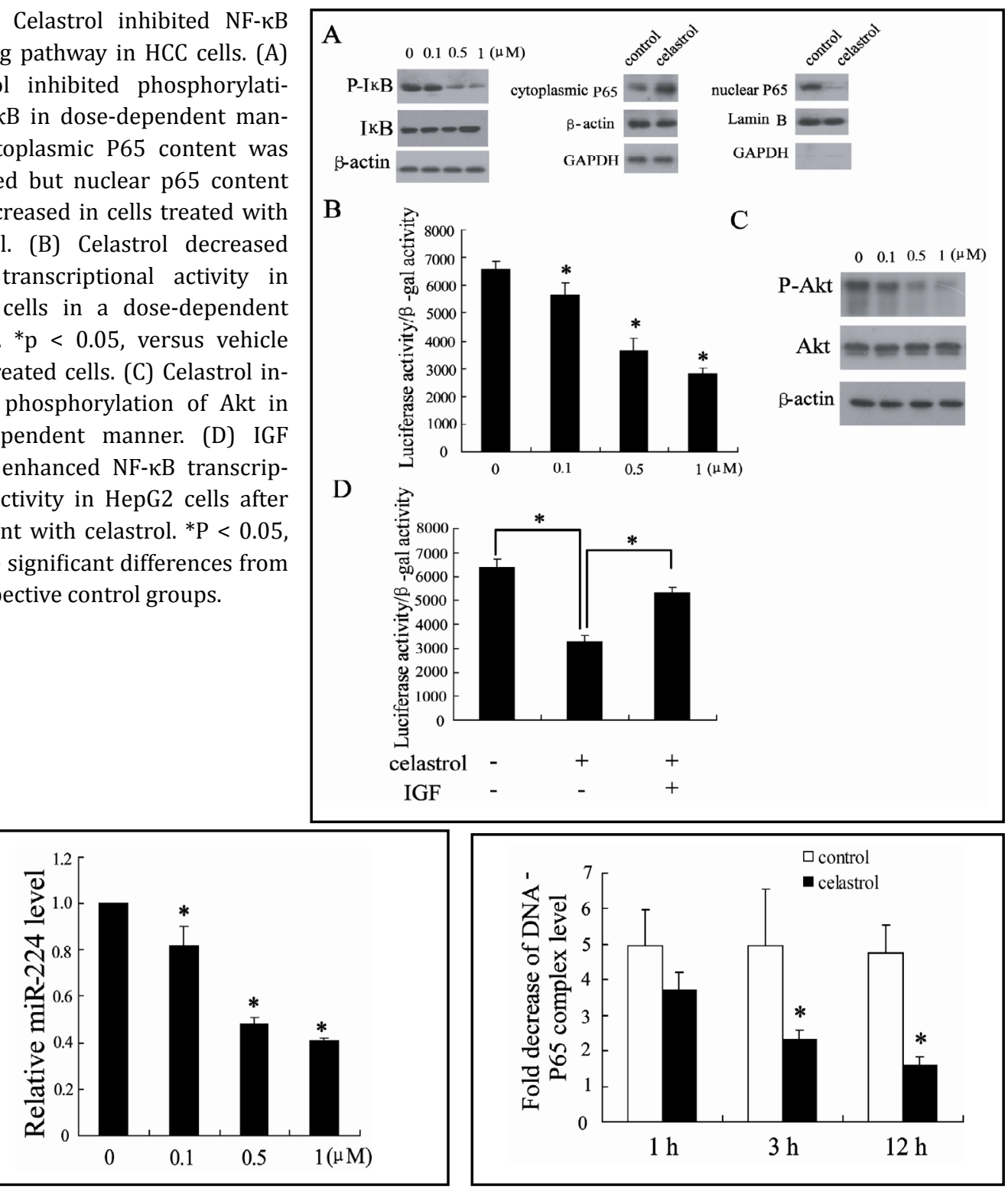

Fig. 3. Celastrol inhibits miR-224 expression in HCC cells. ${ }^{*} \mathrm{p}<0.05$, versus vehicle alone-treated cells.

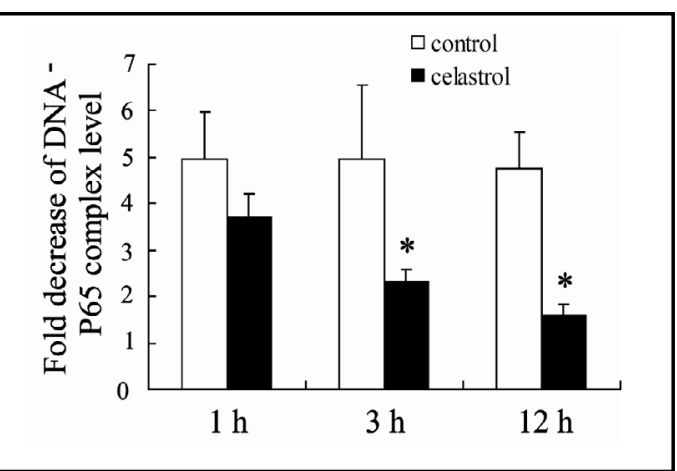

Fig. 4. Celastrol treatment resulted in alteration of p65/NF- $\kappa \mathrm{B}$ binding the miR-224 promoter. The capacity of $\mathrm{p} 65 / \mathrm{NF}-\mathrm{\kappa B}$ binding the miR-224 promoter was determined using ChIP-qPCR analysis in HepG2 cells treated with celastrol $(5 \mu \mathrm{M})$ at indicated times. ${ }^{*} \mathrm{p}<0.05$, versus vehicle alone-treated cells.

The effects of celastrol on miR-224 expression in HCC cells

To further identify the mechanism for celastrol inhibition of migration and invasion of HepG2 cells, we performed real-time PCR to detect miR-224 expression after treated with different concentration of celastrol for $10 \mathrm{~h}$. As shown in Fig. 3, celastrol treatment dosedependently decreased the expression of miR-224 in HepG2 cells $(P<0.01)$.

The effects of celastrol on $p 65 / N F-\kappa B$ binding the miR-224 promoter

To explore whether celastrol could affect p65/NF- $\mathrm{kB}$ transcriptional activity on the miR-224 promoter, ChIP-qPCR assay was performed by using specific primers. The results 
Fig. 5. Celastrol decreased MMP-2 and MMP-9 expression in HepG2 cells. (A) Celastrol had no effect on MMP-2 and MMP-9 mRNA levels. (B) Celastrol decreased MMP-2 and MMP-9 protein levels in a dose-dependent manner. (C) HepG2 cells were treated with celastrol for 12 $h$, and then MG132 was added for an additional $4 \mathrm{~h}$ before proteins were harvested to perform western blot analysis.

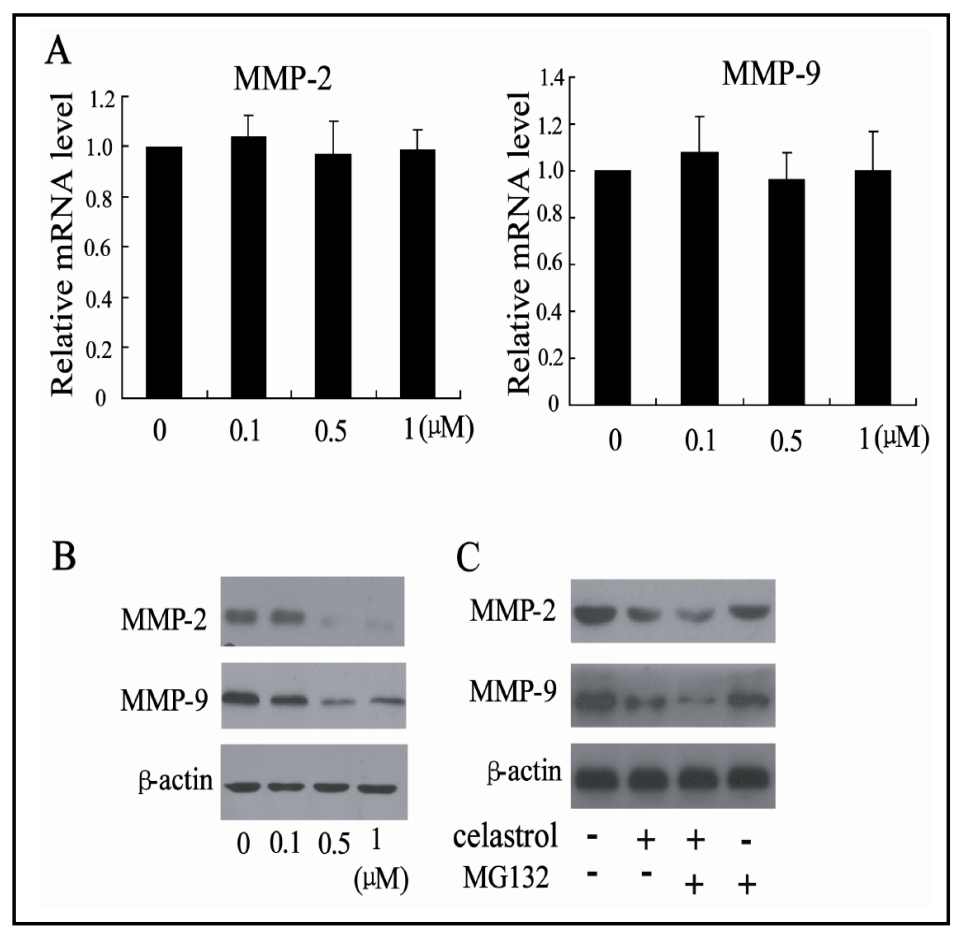

Fig. 6. Down-regulation of miR224 expression can inhibit MMP-2 and MMP-9 expression in HepG2 cells. (A) The real-time PCR revealed that miR-224 inhibitor significantly decreased the expression of miR-224. (B) Western blot showed that miR-224 inhibitor decreased MMP-2 and MMP-9 protein levels. ${ }^{*} \mathrm{P}<0.05$, versus negative control-transfected cells.

A

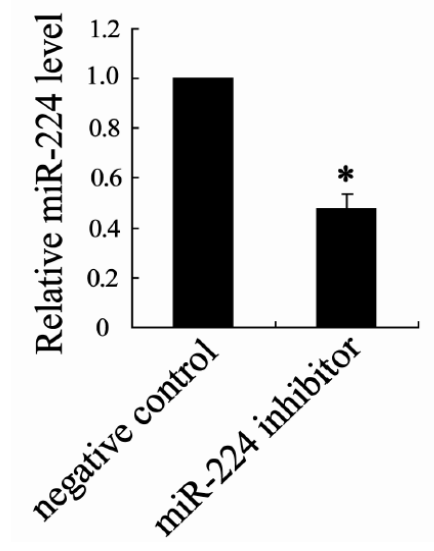

B

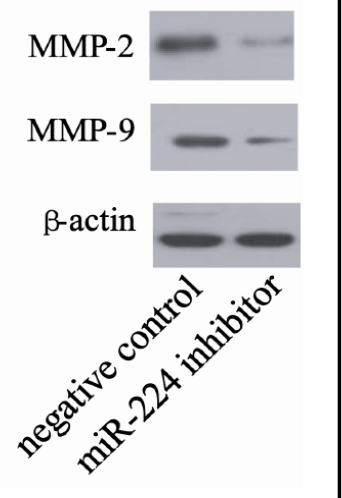

showed that DNA fragments pulled down by the anti-P65 antibody in the celastrol treatment group for $1 \mathrm{~h}, 3 \mathrm{~h}$ and $12 \mathrm{~h}$ were reduced to approximately 0.1 -fold, 0.45 -fold and 0.67 -fold, respectively, for the miR-224 promoter site (Fig. 4). Additionally, in the control groups, DNA fragments pulled down by anti-P65 antibody were 3.5 times higher than those pulled down by IgG for the miR-224 promoter site.

The effects of celastrol on MMPs expression in HCC cells

It is well known that MMPs have been important components of cancer invasion [23]. So we examined the influence of celastrol stimulation on MMP-2 and MMP-9 activity in HepG2 cells. The results of mRNA level were assayed by realtime PCR and were shown in Fig. 5A.We found that MMPs mRNA levels are NOT altered by celastrol treatment.

In order to determine the MMP- 2 and MMP-9 protein levels in cells after treatment with celastrol, we performed western blot and the result was shown in Fig. 5B. Inconsistent with the observed alteration of their mRNA levels, MMP-2 and MMP-9 protein levels were significantly reduced in a dose-dependent manner after treatment with celastrol. 
Fig. 7. Up-expression of miR-224 can reverse the effect of celastrol on MMP-2 and MMP-9 expression as well as migration and invasion. (A) The real-time PCR revealed that miR-224 precursor can significantly increase the expression of miR224 in HepG2 and Huh7 cells. (B) After transfected with $100 \mathrm{nmol} / \mathrm{L}$ of miR-224 precursor, the cells were treated with $0.5 \mu \mathrm{M}$ celastrol. miR-224 precursor significantly increased MMP-2 and MMP-9 expression in HepG2 cells and Huh7 with or without celastrol treatment. miR-224 precursor significantly increased migration ability in HepG2 cells after celastrol treatment. ${ }^{* P}<$ 0.05 , indicate significant differences

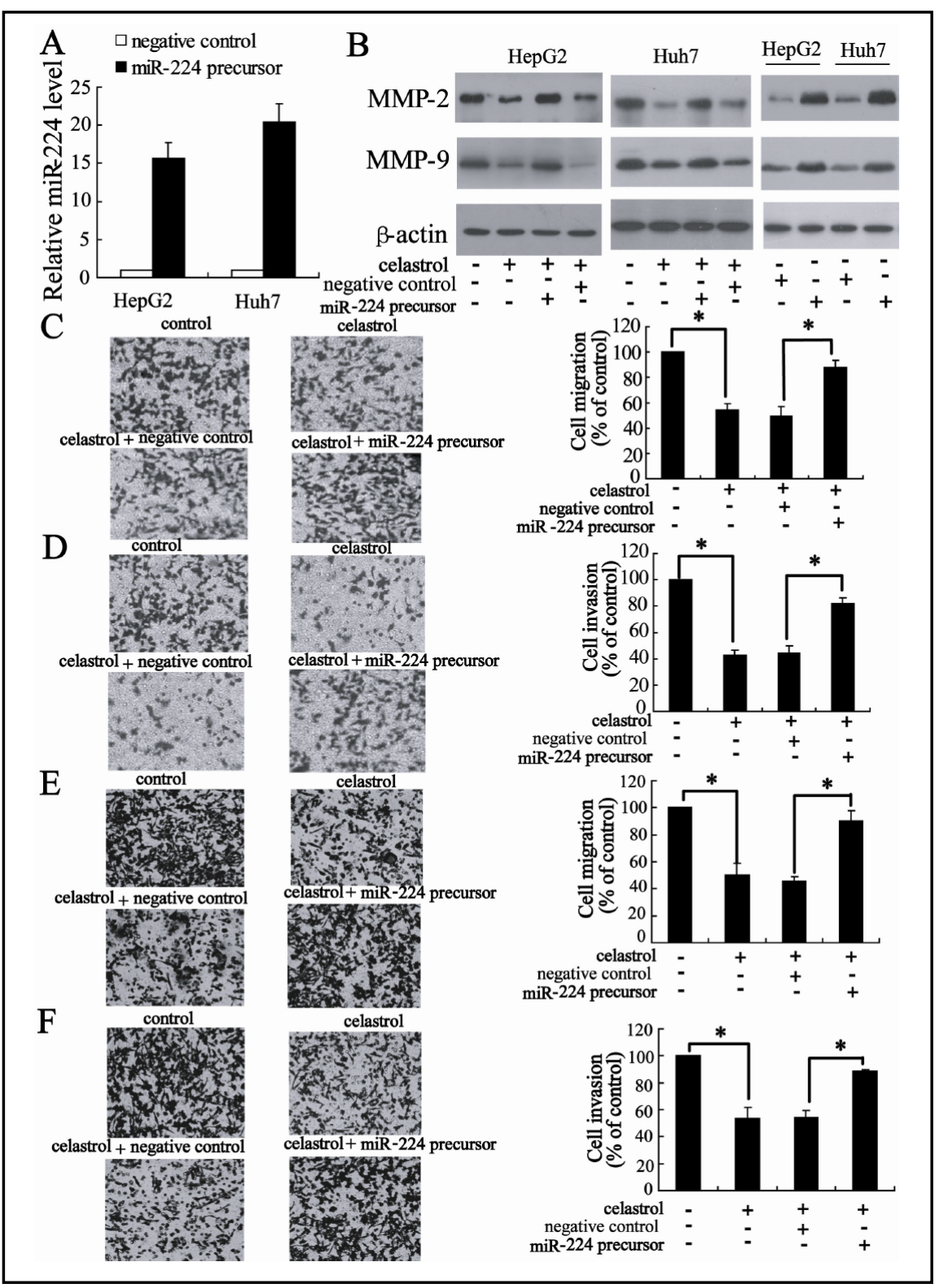

from the respective control groups. (D) miR-224 precursor significantly increased invasion ability in HepG2 cells after celastrol treatment. ${ }^{*} \mathrm{P}<0.05$, indicate significant differences from the respective control groups. (E) miR-224 precursor significantly increased migration ability in Huh7 cells after celastrol treatment. ${ }^{*} \mathrm{P}<0.05$, indicate significant differences from the respective control groups. (F) miR-224 precursor significantly increased invasion ability in Huh7 cells after celastrol treatment. ${ }^{*} \mathrm{P}<0.05$, indicate significant differences from the respective control groups.

\section{The effects of miR-224 on MMPs expression in HCC cells}

To determine whether miR-224 can regulate signaling pathway in HCC cells, we investigated MMP-9 activity and expression in cells transfected miR-224 inhibitor. The result of real-time PCR revealed that miR-224 inhibitor can significantly decrease the expression of miR-224 in HepG2 cells $(P<0.01)$ (Fig. 6A), suggesting that miR-224 inhibitor is efficiently introduced into the cells and acts to down-regulate miR-224 expression.

The result of western blot showed that miR-224 inhibitor reduced MMP-2 and MMP-9 expression in HepG2 cells (Fig. 6B). 
The effect of celastrol on MMPs expression and the migration and invasion of HCC cells with miR-224 precursor

It has been proved that MicroRNA-224 is upregulated in HCC cells and involved in cellular migration and invasion $[9,10]$. In order to evaluate the role of miR-224 in the effect of celastrol on inhibition of HCC cells migration and invasion ability, we used miR-224 precursor. As shown in Fig. 7A, the real-time PCR revealed that miR-224 precursor significantly increased the expression of miR-224, suggesting that miR-224 precursor is efficiently introduced into HepG2 and Huh7 cells and acts to overexpress miR-224. In addition, we found that miR224 precursor can reverse MMP-2 and MMP-9 expression inhibited by celastrol in HepG2 and Huh7 cells (Fig. 7B). We also found that miR-224 precursor could stimulate MMP-2 and MMP-9 expression in HepG2 and Huh7 cells without celastrol treatment. More importantly, miR-224 precursor can reverse migration and invasion ability of cells after treatment with celastrol (Fig. 7C, 7D, 7E and 7F).

\section{Discussion}

Celastrol, a plant triterpene, is of particular interest because of its anti-tumor capabilities of inhibiting the proliferation, motility, and invasiveness of cancer cells [24]. Although several reports mentioned about the involvement of celastrol in apoptosis of hepatoma cells, no study has been reported the anti-metastatic function of celastrol in HCC [25, 26]. In this study, we investigated the effect of celastrol on migration and invasion ability of HCC cells. Treatment with celastrol inhibits migration and invasion of HepG2 cells, and NF- $\kappa B$ and Akt activity. Besides, celastrol stimulation efficiently decreased miR-224, MMP-2 and MMP-9 expression. Furthermore, down-regulation of miR-224 expression can inhibit MMP-2 and MMP-9 expression. So we speculate that the effect of celastrol on inhibition of migration and invasion ability is probably due to the inhibition of miR-224 expression. We further found miR-224 precursor can reverse the effect of celastrol on inhibition migration and invasion ability of HepG2 and Huh7 cells.

Migration and invasion of the extracellular matrix are central steps in tumor metastasis. MMP-2 and MMP-9 can degrade ECM components and play important role in hepatocellular carcinoma migration and invasion [27]. Kim et al reported that celastrol stimulation decreased MMP-9 transcription via NF- $\mathrm{KB}$ signaling pathway in breast cancer cells [17]. We also found that celastrol significantly inhibited the expression of MMP-2 and MMP-9 at the protein levels but had no effect on mRNA expression in HepG2 cells. As a result, the inhibitory effect of celastrol on the MMP-9 expression is not due to NF- $\kappa B$ directly regulating MMP-9 gene transcription.

To further clarify the mechanism involved in the inhibition of MMP-2 and MMP-9 expression of HepG2 cells, we investigated the effect of celastrol on expression of miR-224. Previous studies showed that miR-224 is highly expressed in HCC tissues and cells, and involved in migration and invasion by stimulating MMP-9 expression [10]. Our data revealed that celastrol down-regulated the expression of miR-224 in dose-dependent manner. Most importantly, we found that the overexpression of miR-224 could restore the invasive capacity of HepG2 and Huh7 cells inhibited by celastrol, suggesting that miR-224 was indeed involved in invasion inhibition induced by celastrol. When miR-224 expression was suppressed, the protein levels of MMP-2 and MMP-9 was significantly decreased. Taken together, these data suggest that celastrol exerted antimetastatic effect through miR-224 down-regulation of MMP-2 and MMP-9 expression. However, more clarification of the specific mechanism of miR-224 regulating MMP-2 and MMP-9 expression is still needed.

P65/NF- $\kappa$ B is a direct transcriptional regulator of miR-224 expression and link miR224 with cell migration and invasion in HCC [11]. Here, we showed that under celastrol treatment, the decrease of miR-224 expression level was accompanied by the reduction of activity of p65/NF- $\kappa B$ binding the miR-224 promoter. In the control group, we found that DNA fragments pulled down by anti-P65 antibody were 3.5 times higher than those pulled 
Li/Li/Liu/Sun/Yang/Liu : Celastrol on Migration and Invasion of HCC Cells

down by IgG for the miR-224 promoter site. These data revealed that constitutive NF- $\kappa B$ activation stimulated miR-224 expression, which provided clues to explain the mechanism of miR-224 up-regulation in HCC.

It has been reported that there are crosstalk between PI3K and NF- $\kappa$ B signaling pathway in a wide variety of human cancers [28]. Consistent with the above reported, our results showed that celastrol can inhibit NF- $\mathrm{kB}$ transcriptional activity in a PI3K/Akt-dependent manner. As shown in Fig. 2C, treatment with celastrol led to inactivation of Akt in HepG2 cells. Moreover, IGF-1 reversed the inhibitory effects of celastrol on NF- $\kappa \mathrm{B}$ activity. These data suggest that suppression of the PI3K/Akt pathway is involved in celastrol-inhibited NF$\kappa \mathrm{B}$ transcriptional activity.

In conclusion, we found that celastrol could suppress Akt and NF- $\kappa \mathrm{B}$ activity, which in turn downregulate the expression of miR-224. Down-regulation of miR-224 expression resulted in the decrease of MMP-2 and MMP-9 expression as well as inhibition of migration and invasion in HCC cells. These results suggest that further in vivo studies and clinical trials are needed to establish whether celastrol could be an effective drug for HCC ideal.

\section{Acknowledgements}

This work was supported by grants from the National Natural Science Foundation of China (81071955) and Scientific Research from Educational Department of Liaoning Province, China (L2010634) to H. Y. Li.

\section{Conflict of Interest}

None

\section{References}

1 Venook AP, Papandreou C, Furuse J, de Guevara LL: The Incidence and Epidemiology of Hepatocellular Carcinoma: A Global and Regional Perspective. Oncologist 2010;15:5-13.

-2 Ribatti D, Vacca A, Nico B, Sansonno D, Dammacco F: Angiogenesis and anti-angiogenes is in hepatocellular carcinoma. Cancer Treat Rev 2006;32:437-444.

3 Pang RW, Joh JW, Johnson PJ, Monden M, Pawlik TM, Poon RT: Biology of hepatocellular carcinoma. Ann Surg Oncol 2008;15:962-971.

-4 Okazaki I, Inagaki Y: Novel strategies for hepatocellular carcinoma based on MMPs science. Anticancer Agents Med Chem 2012;12:753-763.

-5 Inui M, Martello G, Piccolo S: MicroRNA control of signal transduction. Nat Rev Mol Cell Biol 2010;11:252263.

6 Miska EA: How microRNAs control cell division, differentiation and death. Curr Opin Genet Dev 2005;15:563-568.

7 Davis-Dusenbery BN, Hata A: MicroRNA in cancer: the involvement of aberrant microRNA biogenesis regulatory pathways. Genes Cancer 2010;1:1100-1114.

8 Nicoloso MS, Spizzo R, Shimizu M, Rossi S, Calin GA: MicroRNAs - the micro steering wheel of tumour metastases. Nat Rev Cancer 2009;9:293-302.

-9 Zhang Y, Takahashi S, Tasaka A, Yoshima T, Ochi H, Chayama K: Involvement of microRNA-224 in cell proliferation, migration, invasion, and anti-apoptosis in hepatocellular carcinoma. J Gastroenterol Hepatol 2013;28:565-575.

10 Li Q, Wang G, Shan JL, Yang ZX, Wang HZ, Feng J, Zhen JJ, Chen C, Zhang ZM, Xu W, Luo XZ, Wang D: MicroRNA-224 is upregulated in HepG2 cells and involved in cellular migration andinvasion. J Gastroenterol Hepatol 2010;25:164-171. 
Li/Li/Liu/Sun/Yang/Liu : Celastrol on Migration and Invasion of HCC Cells

- 11 Scisciani C, Vossio S, Guerrieri F, Schinzari V, De Iaco R, D'Onorio de Meo P, Cervello M, Montalto G, Pollicino T, Raimondo G, Levrero M, Pediconi N: Transcriptional regulation of miR-224 upregulated in human HCCs by NFKB inflammatory pathways. J Hepatol 2012;56:855-861.

12 Salminen A, Lehtonen M, Paimela T, Kaarniranta K: Celastrol: Molecular targets of Thunder God Vine Biochem Biophys Res Commun 2010;394:439-442.

13 Morita T: Celastrol: a new therapeutic potential of traditional Chinese medicine. Am J Hypertens 2010;23:821.

14 Kannaiyan R, Shanmugam MK, Sethi G: Molecular targets of celastrol derived from Thunder of God Vine: potential role in the treatment of inflammatory disorders and cancer. Cancer Lett 2011;303:9-20.

15 Kannaiyan R, Manu KA, Chen L, Li F, Rajendran P, Subramaniam A, Lam P, Kumar AP, Sethi G: Celastrol inhibits tumor cell proliferation and promotes apoptosis through the activation of c-Jun N-terminal kinase and suppression of PI3 K/Akt signaling pathways. Apoptosis 2011;16:1028-1041.

16 Zhu H, Liu XW, Cai TY, Cao J, Tu CX, Lu W, He QJ, Yang B: Celastrol acts as a potent antimetastatic agent targeting beta1 integrin and inhibiting cell-extracellular matrix adhesion, in part via the p38 mitogenactivated protein kinase pathway. J Pharmacol Exp Ther 2010;334:489-499.

17 Kim Y, Kang H, Jang SW, Ko J: Celastrol inhibits breast cancer cell invasion via suppression of NF-kBmediated matrixmetalloproteinase-9 expression. Cell Physiol Biochem 2011;28:175-184.

18 Vermes I, Haanen C, Reutelingsperger C: Flow cytometry of apoptotic cell death. J Immunol Methods 2000;243:167-190.

19 Gong F, Sun L, Wang Z, Shi J, Li W, Wang S, Han X, Sun Y: The BCL2 gene is regulated by a special AT-rich sequence binding protein 1-mediated long range chromosomal interaction between the promoter and the distal element located within the 3'-UTR. Nucleic Acids Res 2011;39:4640-4652.

20 Mukhopadhyay A, Deplancke B, Walhout AJ, Tissenbaum HA: Chromatin immunoprecipitation (ChIP) coupled to detection by quantitative real-time PCR to study transcription factor binding to DNA in Caenorhabditis elegans. Nat Protoc 2008;3:698-709.

21 Rajendran P, Li F, Shanmugam MK, Kannaiyan R, Goh JN, Wong KF, Wang W, Khin E, Tergaonkar V, Kumar AP, Luk JM, Sethi G: Celastrol suppresses growth and induces apoptosis of human hepatocellular carcinoma through the modulation of STAT3/JAK2 signaling cascade in vitro and in vivo. Cancer Prev Res (Phila) 2012;5:631-643.

22 Li J, Lau G, Chen L, Yuan YF, Huang J, Luk JM, Xie D, Guan XY: Interleukin 23 promotes hepatocellular carcinoma metastasis via NF-kappa B induced matrix metalloproteinase 9 expression. PLoS One 2012; 7:e46264.

23 Johansson N, Ahonen M, Kähäri VM: Matrix metalloproteinases in tumor invasion. Cell Mol Life Sci 2000;57:5-15.

-24 Gupta SC, Kim JH, Prasad S, Aggarwal BB: Regulation of survival, proliferation, invasion, angiogenesis, and metastasis of tumor cellsthrough modulation of inflammatory pathways by nutraceuticals. Cancer Metastasis Rev 2010;29:405-434.

25 Zhu H, Yang W, He LJ, Ding WJ, Zheng L, Liao SD, Huang P, Lu W, He QJ, Yang B: Upregulating Noxa by ER stress, celastrol exerts synergistic anti-cancer activity in combination with ABT-737 in human hepatocellular carcinoma cells. PLoS One 2012;7:e52333.

26 Zhang D, Xu L, Cao F, Wei T, Yang C, Uzan G, Peng B: Celastrol regulates multiple nuclear transcription factors belonging to HSP90's clients in a dose- and cell type-dependent way. Cell Stress Chaperones 2010;15:939-946.

27 Ogasawara S, Yano H, Momosaki S, Nishida N, Takemoto Y, Kojiro S, Kojiro M: Expression of matrix metalloproteinases (MMPs) in cultured hepatocellular carcinoma (HCC) cells and surgically resected HCC tissues. Oncol Rep 2005;13:1043-1048.

28 Rasul A, Ding C, Li X, Khan M, Yi F, Ali M, Ma T: Dracorhodin perchlorate inhibits PI3K/Akt and NF-кB activation, up-regulates the expression of p53, and enhances apoptosis. Apoptosis 2012;17:1104-1119. 